\title{
Non-invasive monitoring is coming the full circle, making our patients safer!
}

\author{
Michael A. Ramsay ${ }^{1}$ (D)
}

Received: 8 January 2020 / Accepted: 14 January 2020 / Published online: 18 January 2020

(c) Springer Nature B.V. 2020

\begin{abstract}
Non-invasive monitoring is becoming more accurate, more available and mobile. The clinical advantage that this developing technology provides is that the data may be monitored continuously; relatively unobtrusively, and transmitted directly to the caregiver. The downside of being non-invasive has been the potential loss of accuracy in the data displayed. This has been overcome in the measurement of oxygen saturation of hemoglobin by pulse oximetry, in that treatment will be instigated by a decline in oxygen saturation without necessarily an arterial blood gas analysis being performed. The development of pulse oximetry to measure hemoglobin levels ( $\mathrm{SpHb}$ ) has relied on "trend accuracy" to indicate the need for a confirmatory laboratory analysis of hemoglobin level. The study by Applegate et al. [1] confirms the trend accuracy of SpHb as an indication to perform a laboratory confirmation of hemoglobin level. This will lead to earlier laboratory screening, so that developing adverse conditions, such as postoperative bleeding, may be identified at a time that major events, such as failure to rescue can be avoided. This increased availability of non-invasive technology will make patients safer both in our hospitals and at home.
\end{abstract}

Keywords Noninvasive monitoring · Improved surveillance technology · Patient safety · Reducing "Failure to Rescue" events

In this edition of JCMC, Applegate et al. demonstrate that the improvement in technology in pulse oximetry is advancing the trend accuracy of non-invasive hemoglobin $(\mathrm{SpHb})$ measurement [1]. This will hopefully lead to the end of the "stat" bring back to the operating room at 7 am in the morning of patients, when the routine morning laboratory results are reviewed. The non-invasive monitoring of $\mathrm{SpHb}$ may have demonstrated the declining $\mathrm{SpHb}$ at midnight causing a laboratory hemoglobin check and either the treatment of a coagulopathy negating the need for a return to the operating room or an early re-exploration. The continuous display of $\mathrm{SpHb}$ may lead to the more appropriate blood management of major surgical patients by indicating when a laboratory check is necessary.

When I started practicing anesthesia 40 years ago. Noninvasive monitoring was the norm for most patients undergoing anesthesia. This consisted of a finger on the pulse, that

Michael A. Ramsay

michael.ramsay@bswhealth.org

1 Department of Anesthesiology, Baylor University Medical Center, Baylor Scott and White Health Care System, Dallas, TX, USA measured the strength, volume and character of the pulse continually. If you can feel the superficial temporal pulse the blood pressure is at least $70 \mathrm{~mm}$ hg was the assumption. Assessment of temperature and diaphoresis was made by observation and touch of the forehead. A monoaural stethoscope, with custom made earpiece, allowed assessment of breath sounds, respiratory rate and heart sounds. A pump sphygmomanometer was placed on some patients with a stethoscope under the cuff, and the eyes were not taped so that the pupils could be observed for size and reactivity, as a guide to depth of anesthesia. However, the assessment of oxygenation was only made by the physical appearance of the patient, which meant determining if they were pink or cyanotic, a difficult task in the dark operating room!

The development of pulse oximetry has been one of the most important advances in anesthesia. The measurement of oxygenation has been investigated for centuries by scientists. The Lambert-Beer Law was developed in the nineteenth Century and described the attenuation of light to the properties of the material through which the light is travelling [2]. This law is the basis for the development of oximetry. Around the same time a German physiologist Felix HoppeSeyler applied absorption spectroscopy to hemoglobin. This 
is based on the principle that solutions are colored because they absorb and reflect specific wavelengths of light. He demonstrated in 1864 that if light passed through a solution of oxygenated hemoglobin, wavelengths $540 \mathrm{~nm}$ and $560 \mathrm{~nm}$ would be absorbed, twin — peak absorption pattern [3]. A British physicist Stokes applied this research to the changing color of blood with deoxygenation, and found that the twin-peak changed to a single peak as the hemoglobin desaturated. This resulted in the understanding that oxygen was carried in the blood by hemoglobin. Many further developments took place over the first half of the twentieth century but it was a Japanese bioengineer Takuo Aoyagi who became interested in oximetry and realized that the pulsations of arterial blood could be used to measure arterial oxygen saturation without a zero calibration in a bloodless sample. This put the word pulse into oximetry and he used more precise wavelengths of light to detect absorption of oxygen by oxyhemoglobin $[4,5]$.This technology was very sensitive to motion but demonstrated that the principle was accurate. Within 10 years of Aoyagi's invention, pulse oximetry was introduced at the American Society of Anesthesiologists Annual meeting by William New MD and Mark Yelderman MD and published in the journal Anesthesiology [6].

In 1989 an electrical engineer Joe Kiani, who was later joined by another engineer Mohamed Diab, developed measure-through motion and low perfusion pulse oximetry. [8] This led to further advances where more than seven wavelengths of light allow the continuous and noninvasive measurement of hemoglobin $(\mathrm{SpHb})$ carboxyhemoglobin (SpCO), and methemoglobin (SpMet), in addition to oxygen saturation (SpO2), pulse rate, perfusion index, $(\mathrm{Pi})$ and pleth variability index (PVi) [7-9].

Now with enhanced technology and increasing accuracy, non-invasive monitoring is becoming more available both for personal and clinical use. Wearable devices that have wireless communication to caregivers' smart phones offer an improved safety margin that has the potential of eliminating "Failure to Rescue" in our hospitals, as clinical deterioration will be detected at a reversible time.

In my career, I started with inaccurate noninvasive applications, then monitoring became very invasive in the drive for accuracy, but saw many comorbidities associated with this strategy. Now we are in the era of more accurate, noninvasive technology that is mobile and transmittable and will lead to more timely patient management and increased patient safety.

Funding This author received no funding from any source in the preparation or content of this editorial.

\section{Compliance with ethical standards}

Conflict of interest I have received Research Grants from Masimo Corp.

Research involving human and/or animal participants This editorial does not contain any studies with human participants or animals performed by the author.

\section{References}

1. Applegate Ii RL, Applegate PM, Cannesson M, Peiris P, Ladlie BL, Torp K. Multicenter comparison of three intraoperative hemoglobin trend monitoring methods. J Clin Monit Comput. 2019. https://doi.org/10.1007/s10877-019-00428-3.

2. Van Meter A, Williams U, Zavala A, Kee J, Rebello E, Tsai J, Ifeanyi I, Ruiz J, Owusu-Agyemang P. Beat to Beat: a measured Look at the History of Pulse Oximetry. J Anesth Hist. 2017;3:24-6.

3. Hoppe F. Uber die chemischen und optischen Eigenschaften des Blutforbstoffs. Arch Pathol Anat Physiol. 1864;29:233-5.

4. Aoyagi T. Pulse oximetry: its invention, theory and future. J Anesth. 2003;17:259-66.

5. Severinghaus JW, Aoyagi T. Discovery of pulse oximetry. Anesth Analg. 2007;105(6):S1-S4.

6. Yelderman M, New W. Evaluation of pulse oximetry. Anesthesiology. 1983;4:349-52.

7. Barker SJ, "Motion-Resistant" Pulse Oximetry: A Comparison of New and Old Models. Ameth Analg 2007;95:967-72

8. Barker SJ, Badel JJ. The measurement of dyshemoglobins and total hemoglobin by pulse oximetry. Curr Opin Anesthesiol. 2008;21:805-10.

9. Forget P, Lois F, De Kock M. Goal-directed fluid management based on the pulse oximeter-derived pleth variability index reduceslactate levels and improves fluid management. Anesth Analg 2010;111:910-14

Publisher's Note Springer Nature remains neutral with regard to jurisdictional claims in published maps and institutional affiliations. 